\title{
Impact actuel et futur des écosystèmes terrestres sur notre climat
}

\author{
Bernard LONGDOZ et Bernard HEINESCH
}

Université de Liège, Gembloux Agro-Bio Tech, TERRA Teaching and Research Center (BIODYNE team)

\section{Introduction}

Le changement climatique est dû à une captation de plus en plus importante de l'énergie solaire par notre Terre. Les chercheurs et chercheuses ont mis en évidence que cette capture énergétique additionnelle de $2,29 \mathrm{~W} / \mathrm{m}^{2}$ depuis 1750 , appelée aussi forçage radiatif, résulte essentiellement de l'augmentation de la concentration atmosphérique de trois gaz à effet de serre $(\mathrm{GES})^{[1]}$ (le dioxyde de carbone $\mathbf{C O}_{2}$ responsable d'un forçage de $1,68 \mathrm{~W} / \mathrm{m}^{2}$, le méthane $\mathbf{C H}_{4}$ avec $0,97 \mathrm{~W} / \mathrm{m}^{2}$ et le protoxyde d'azote $\mathbf{N}_{\mathbf{2}} \mathbf{O}$ pour $0,17 \mathrm{~W} / \mathrm{m}^{2}$, auxquels s'ajoutent les forçages positifs et négatifs d'autres processus d'origine anthropique). Ces valeurs peuvent sembler faibles mais sont suffisantes pour provoquer les évolutions rapides du climat que nous constatons déjà actuellement. Les études scientifiques se sont alors focalisées sur les flux de ces gaz à effet de serre pour comprendre comment on pourrait limiter cette augmentation et préserver notre climat. Il est vite apparu que notre consommation énergétique (industrie, transport, chauffage et refroidissement) était la principale source mais que les écosystèmes terrestres comme par exemple les forêts, les prairies ou les cultures étaient aussi des acteurs importants (comme puits ou sources) au même titre que les océans. A côté de ce rôle, les écosystèmes agissent aussi sur le climat par d'autres vecteurs tel que leur pouvoir réfléchissant pour le rayonnement solaire, leur faculté de freinage du vent, leur émission de gaz réactifs qui vont modifier la composition atmosphérique ou leur fonction dans le cycle hydrologique (transpiration) ${ }^{[2]}$. L'ensemble de ces activités ne seront pas abordées dans cet article qui sera consacré à la contribution des écosystèmes terrestres dans la modification de l'effet de serre.

\section{Forêts, prairies et cultures : des acteurs importants dans l'effet de serre}

Les écosystèmes terrestres nous rendent plusieurs services comme la fourniture de nourriture, de produits sylvicoles (bois de construction, de chauffage, papier...), la filtration et le stockage de l'eau, le maintien de la biodiversité, la lutte contre l'érosion ou la perte de fertilité des sols ${ }^{[3]}$. Ils constituent aussi un endroit de stockage pour le carbone (C) en provenance de l'atmosphère. Ensemble, ils constituent un puits important puisqu'ils captent $29 \%$ du $\mathrm{CO}_{2}$ émis par les activités humaines ${ }^{[4]}$. Par contre, les prairies pâturées (lieu d'élevage de ruminants producteurs de méthane), les écosystèmes humides (wetlands) et la fermentation des produits organiques de culture non consommés constituent la source majoritaire de $\mathrm{CH}_{4}$ sur notre planète ${ }^{[5]}$. En ce qui concerne le $\mathrm{N}_{2} \mathrm{O}$, les évaluations sont moins précises, mais on 
estime que les cultures et les pâtures représentent plus de la moitié des sources suite à leur fertilisation azotée d'origine synthétique ou organique (liée aux excrétas) ${ }^{[6]}$.

$\mathrm{Au}$ vu de ces pourcentages, on s'aperçoit qu'il est important de mieux comprendre les mécanismes impliqués dans les échanges de GES entre les écosystèmes et l'atmosphère pour proposer une gestion de nos écosystèmes diminuant les émissions de méthane et de protoxyde d'azote et augmentant le captage du $\mathrm{CO}_{2}$. Cependant, les changements climatiques vont aussi influencer ces flux ce qui peut parfois entrainer une rétroaction tendant à renforcer le changement climatique (par exemple l'augmentation des températures va amplifier la respiration des organismes et donc la production de $\mathrm{CO}_{2}$ ).

Nous vous proposons dans un premier temps d'identifier les processus à l'origine des flux de GES échangés par les écosystèmes terrestres et de passer en revue les déterminants biotiques et abiotiques de ces flux. Ensuite nous examinerons quels modes de gestion semblent les plus adaptés afin de renforcer le rôle atténuateur de ces écosystèmes terrestres pour le forçage radiatif.

\section{Processus liés aux productions et absorptions de GES par les écosystèmes terrestres}

\subsection{Dioxyde de carbone}

En ce qui concerne le $\mathrm{CO}_{2}$, le principal flux est la photosynthèse des chlorophylles des végétaux. Cette photosynthèse s'accompagne systématiquement d'une photo-respiration pour donner une assimilation brute (Gross Primary Productivity, GPP, Fig. 1) d'environ 440 $\mathrm{GtCO}_{2} /$ an à l'échelle de notre planète ${ }^{[4]}$ (soit environ 13 fois les émissions dues à l'utilisation des carburants fossiles). Ce flux peut être mesuré par la méthode des covariances turbulentes (voir section 4). Le rayonnement solaire est la principale variable d'influence sur ce flux (augmentation linéaire jusqu'à un niveau de saturation) mais il peut être réduit par un déficit en eau du sol ou une sécheresse aérienne et accentué par une température ou une concentration de $\mathrm{CO}_{2}$ dans l'air plus élevées. Ces facteurs affectent l'ouverture stomatique par laquelle le $\mathrm{CO}_{2}$ pénètre dans la feuille et/ou la réaction biochimique photosynthétique en ellemême. A quantité de rayonnement identique, une lumière diffuse (avec un spectre à composante bleu plus prononcée) est aussi plus favorable à l'absorption de $\mathrm{CO}_{2}$ tout comme une plus grande surface foliaire par $\mathrm{m}^{2}$ de sol et une concentration élevée en azote foliaire. 


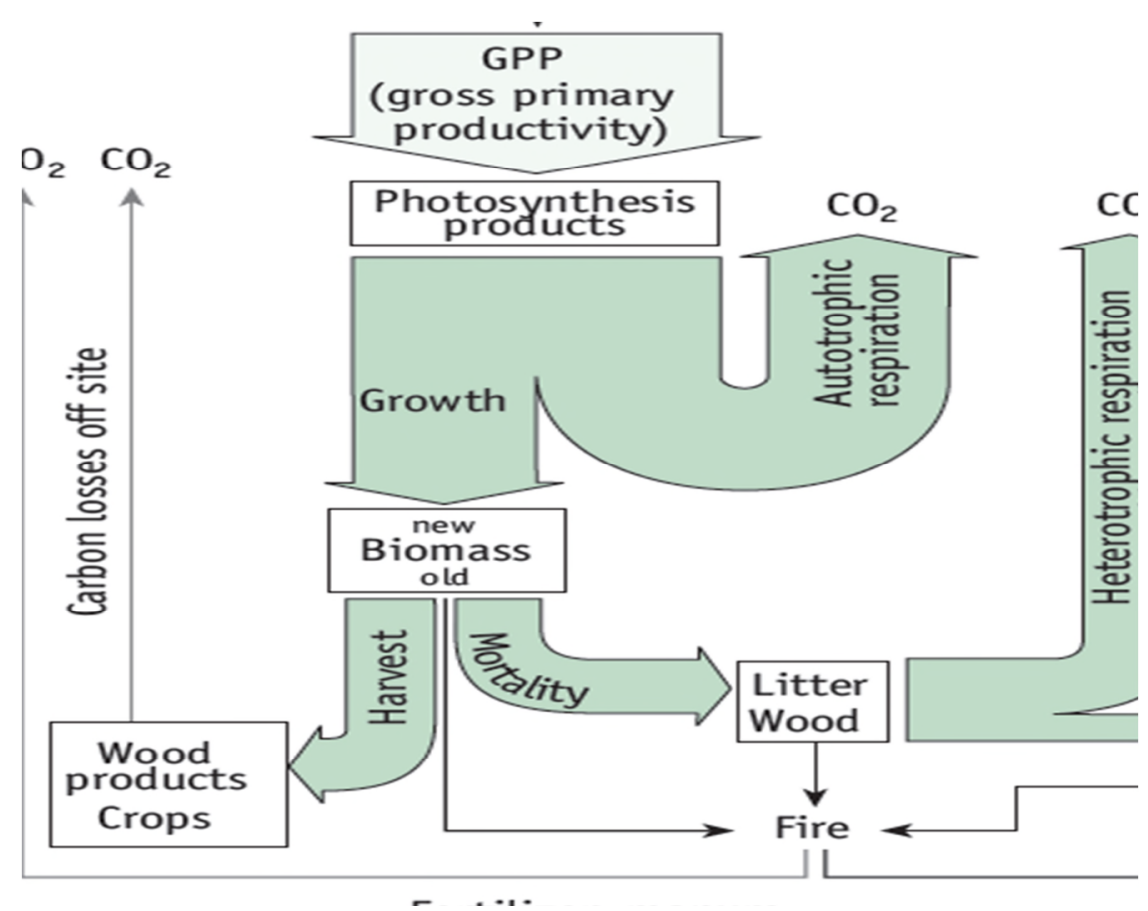

Figure 1 : Cycle du carbone dans les écosystèmes terrestres, centré sur les échanges de $\mathrm{CO}_{2}$ (adapté de Schulze E.-D., 2006, Biological control of the terrestrial carbon sink, Biogeosciences, 2006, 3 (2), pp.147-166.)

Le carbone ainsi assimilé a un temps de résidence très variable dans l'écosystème en fonction de l'utilisation qui en est faite. La grande majorité est assez rapidement respirée (en quelques heures/jours) par les cellules végétales pour être renvoyée vers l'atmosphère. Cette respiration autotrophe étant basée sur une activité enzymatique, elle est typiquement favorisée par une augmentation de la température et est proportionnelle à la biomasse existante. Le reste du carbone est accumulé dans la biomasse sous différentes formes moléculaires organiques et pour une durée de vie très variable. Cela constitue un réservoir variable de $\mathrm{C}$ dans la biomasse qui possède pour flux sortants la stabilisation à très long terme dans les sols, la décomposition sur place ou l'exportation (pour consommation ou stabilisation dans des produits plus pérennes).

Une partie du stock de biomasse peut donc se stabiliser par l'intermédiaire de la formation de litière souterraine (racines mortes et exsudats racinaires) ou aérienne (feuilles et tiges mortes) dont certaines fractions sont fixées dans les sols sous forme de complexes organo-minéraux ou minéraux fossiles. Ce carbone peut être considéré comme soustrait du système atmosphère-écosystèmes-océans. Les autres fractions de litière sont décomposées sur place grâce à l'action des microorganismes du sol qui, en présence d'oxygène, produisent du $\mathrm{CO}_{2}$ (avec une légère augmentation de la biomasse carbonée microbienne). Cette respiration hétérotrophe aérobie est fortement dépendante de la température et de la quantité d'eau dans le sol. En effet l'activité microbienne est croissante avec la température et diminue en cas de sol sec dans lequel peu de carbone dissous est accessible aux microorganismes. À l'opposé, quand la quantité d'eau du sol devient trop importante (tourbières saturées, rizières,...), la décomposition anaérobie produit $\mathrm{du} \mathrm{CH}_{4}$. La respiration hétérotrophe possède aussi une variabilité saisonnière et spatiale liée au type de végétation (composition de la litière) et à la 
phénologie végétale (période et lieu avec ou sans apport de litière) et microbienne (évolution de la biomasse et du type de microorganismes au cours de l'année et entre écosystèmes). La troisième voie de sortie pour la biomasse des écosystèmes est l'exportation pour être utilisée comme aliment, énergie ou composant de produits industriels. En tant que source alimentaire pour l'animal ou l'humain, le $\mathrm{C}$ organique se minéralise en $\mathrm{CO}_{2}$ avec une durée de transit, de sa fixation par photosynthèse jusqu'à sa réémission, allant de quelques jours (pour ce qui est directement assimilé et digéré) à quelques mois ou années (pour ce qui est stocké dans les organismes). Lorsqu'il est utilisé comme carburant de combustion, il possède là aussi une durée de transit vers le $\mathrm{CO}_{2}$ (minéralisation) très variable (de quelques mois pour du miscanthus à plusieurs dizaines d'années pour le bois). Enfin, il est aussi possible de l'utiliser comme matériau entrant dans la composition de différents produits et de le soustraire à moyen terme (papier, bois de trituration), voir à très long terme (construction, nouveaux composés innovants) du système atmosphère-écosystème. L'ensemble des flux d'émission de $\mathrm{CO}_{2}$ (respiration autotrophe et hétérotrophe ainsi que la minéralisation des exportations), représente finalement environ $429 \mathrm{GtCO}_{2}$ /an c'est-à-dire 97,5\% du carbone assimilé par la végétation. Du coup, le bilan net pour ce qui concerne les écosystèmes terrestres est une absorption mais de seulement $11 \mathrm{GtCO}_{2} / \mathrm{an}$ c'est-à-dire $29 \%$ des sources d'origine anthropique et devrait être multipliée par 1,5 pour résorber l'accumulation de $\mathrm{CO}_{2}$ atmosphérique (si le puits océanique conserve la même intensité).

\subsection{Méthane}

Nous avons déjà cité la respiration hétérotrophe anaérobie comme source de méthane dans les écosystèmes terrestres. Elle dépend assez logiquement de la quantité de carbone localisé dans la fraction du sol où l'oxygène est absent (et donc de la hauteur de la nappe phréatique) et est avantagée par des températures élevées. L'autre émission principale de méthane en provenance des écosystèmes terrestres résulte de la fermentation entérique lors de la digestion de nourriture par le bétail. L'ensemble de cette nourriture provient de la production des agrosystèmes (exportation de $\mathrm{C}$ organique pour les prairies et les cultures, citée dans la section précédente). Cette émission est forcément liée à la densité et au type de bétail (capacité de rumination) ainsi qu'à la quantité et au type d'alimentation qui lui est offert. Si la combustion de la biomasse est ajoutée à ces deux sources majeures, l'ensemble représente $60 \%$ des émissions totales, qui s'élèvent à $558 \mathrm{Tg} \mathrm{CH} \mathrm{CH}_{4} / \mathrm{an}^{[5]}$, y compris celles liées à l'utilisation des combustibles fossiles) mais ce méthane émis n'est pas stable dans l'atmosphère d'un point de vue chimique (comme l'est le $\mathrm{CO}_{2}$ ) et une part importante est détruite. En conséquence, ces sources sont 39 fois plus importantes que l'accumulation atmosphérique annuelle qui ne représente que $10 \mathrm{Tg} \mathrm{CH}_{4} / \mathrm{an}$.

\section{$\underline{3.3 \text { Protoxyde d'azote }}$}

Les bilans pour le $\mathrm{N}_{2} \mathrm{O}$ sont beaucoup moins avancés à cause de la difficulté de mesures des flux (voir section 4). Les premières estimations révèlent toutefois que les émissions liées aux 
agrosystèmes (processus de nitrification et dénitrification de l'azote apporté par la fertilisation synthétique ou organique/naturelle) représentent $8,3 \mathrm{Tg} \mathrm{N}_{2} \mathrm{O} / \mathrm{an}$, ce qui correspond à $60 \% \mathrm{du}$ total des émissions. Il n'y a que très peu d'absorption de ce $\mathrm{N}_{2} \mathrm{O}$ par la surface terrestre et la quasi-totalité des émissions s'accumulent dans l'atmosphère

\section{$\underline{3.4 \text { Variabilité des flux net de GES à l'interface atmosphère-écosystème }}$}

$\mathrm{Au}$ vu de ces bilans, nous nous apercevons que les flux nets d'échange écosystèmeatmosphère sont soit très faibles par rapport aux émissions/absorptions (cas du $\mathrm{CO}_{2}$ et du $\mathrm{CH}_{4}$, les deux principaux GES), soit égaux (cas du $\mathrm{N}_{2} \mathrm{O}$ ). Une très faible variation de ces taux d'émission ou d'absorption aura donc une conséquence très importante sur la quantité annuelle de GES qui vient s'accumuler dans l'atmosphère et donc sur l'ampleur de l'effet de serre. Les causes possibles de telles variations sont liées à l'évolution des déterminants des flux cités dans les sections précédentes et que l'on peut regrouper en trois catégories : les conditions de station (type de sol, topographie), les choix de gestion d'un écosystème (espèce végétale, itinéraire technique,...) et le climat (principalement température, rayonnement, contenu en eau du sol et humidité de l'air). Il est donc clair que le contexte de changement climatique et de changement des pratiques agricoles et sylvicoles est de nature à modifier très largement le rôle des écosystèmes vis-à-vis de l'effet de serre, ce qui potentiellement peut renforcer ce dernier.

\section{Comment mesurer les quantités de GES échangées par les écosystèmes}

La mesure des flux de GES peut être réalisée à différentes échelles (organes, parcelles de forêt ou d'agrosystème, région) et doit pour bien faire s'accompagner d'une caractérisation des conditions dans lesquelles ces échanges se sont produits. C'est sur base de ces mesures que des bilans locaux ou globaux peuvent être réalisés et qu'une meilleure compréhension du rôle de chaque déterminant peut être atteinte. Nos recherches se sont focalisées sur le comportement de parcelles entières grâce à la technique de covariance de turbulence (Eddy Covariance, EC), permettant de réaliser un suivi continu du flux de $\mathrm{CO}_{2}$ échangé par une surface homogène de quelques hectares à quelques dizaines d'hectares. Cette technique a été développée à la fin du $20^{\text {ème }}$ siècle, notamment au sein de notre Unité TERRA Teaching and Research Centre, et est basée sur la mesure à haute fréquence (10 à $20 \mathrm{~Hz})$ de la vitesse verticale du vent et de la concentration du traceur étudié, dans la couche limite séparant l'écosystème et l'atmosphère ${ }^{[7]}$. La covariance de ces deux grandeurs permet (après plusieurs opérations en post-traitement) d'obtenir le flux échangé pendant la période d'échantillonnage (typiquement une demi-heure). Comme la prise et l'enregistrement des mesures sont automatisés, les flux peuvent être déterminés pendant toute une année, voire plusieurs années. Le dispositif expérimental est constitué d'un anémomètre ultrasonique à 3 dimensions (mesure de la vitesse verticale du vent), d'un analyseur de gaz par absorption d'infrarouge 
(qui sont localisés quelques mètres au-dessus du sommet de l'écosystème, au sommet d'un mât ou d'une tour) et d'un système d'acquisition et de stockage des données. Il y a quelques années, l'apparition d'analyseurs rapides pour la détermination des concentrations en méthane et en protoxyde d'azote ont permis de réaliser également des mesures de flux nets de ces gaz par EC.

Plusieurs centaines de stations se sont montées à travers le monde sur une multitude d'écosystèmes et sous une large diversité de climats. Elles ont été soutenues par plusieurs projets de recherche pour aboutir assez récemment en Europe à un réseau faisant partie d'un ERIC (European Research Infrastructure Consortium) nommé ICOS (Integrated Carbon Observation System $)^{[8]}$. Le Service Public de Wallonie finance ainsi actuellement une station écosystème labélisée ICOS (une grande culture située à Lonzée près de Gembloux (Fig. 2) et faisant partie de la Cellule «Environment is life » de Gembloux Agro-Bio Tech) et deux autres stations (une prairie pâturée dans le Condroz et une forêt en Ardenne) qui sont en cours de labélisation ${ }^{[9]}$. Ces stations existent depuis plusieurs années et nous ont permis, entre autre, d'établir le bilan annuel en C d'une culture de froment, de betteraves ou de pommes de terre ${ }^{[10]}$. C'est aussi sur base de ces données que nous avons pu analyser le comportement des plantes et du sol lors d'événements climatiques particuliers (sécheresses, vagues de chaleur,...) et aider au paramétrage des flux de GES dans les modèles de fonctionnement des écosystèmes utilisés pour réaliser des études prédictives à large échelle spatiale.

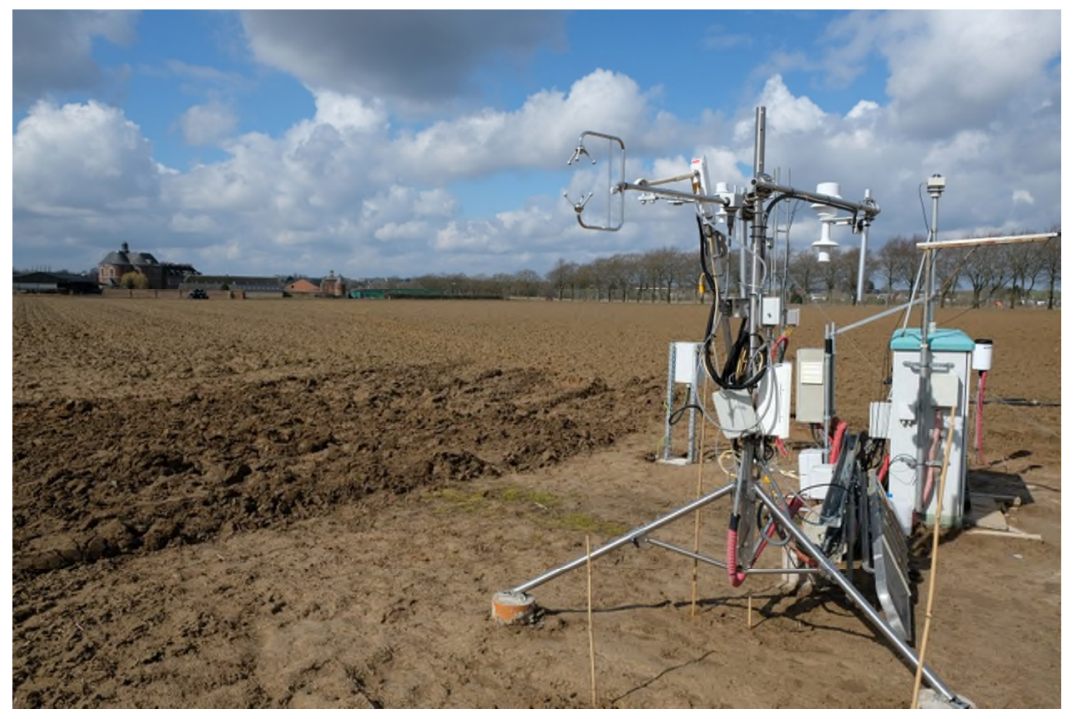

Figure 2 : Dispositif de mesure des flux par covariances de turbulences sur la station ICOS de Lonzée 


\section{L'évolution de ces flux avec le changement climatique}

Comment vont évoluer les échanges de GES lorsqu'ils seront soumis au climat futur engendré par l'augmentation de l'effet de serre ? Les tours à flux ne permettant pas de répondre à cette question autrement que par la modélisation, la Cellule « Environment is life » s'est dotée d'un écotron qui est une infrastructure dans laquelle des mini-parcelles d'écosystèmes $\left(2 \mathrm{~m}^{2}\right)$ se développent sur des lysimètres, grands réservoirs de sol de 1,5 $\mathrm{m}$ de profondeur isolés et sur lesquels on peut faire pousser des plantes. Elles sont enfermées dans des cellules d'environ 50 $\mathrm{m}^{3}$ et sont soumises à un climat complétement contrôlé. Il y a donc moyen de soumettre ces écosystèmes à des conditions (rayonnement, température et humidité de l'air, précipitations, concentration en $\mathrm{CO}_{2}$ et $\mathrm{O}_{3}$, potentiel matriciel hydrique et température du sous-sol) correspondant à un scénario fourni par les climatologues (Representative Concentration Pathway, RCP) ou à des événements extrêmes dont les intensités sont celles prévues dans les années à venir. Le fait de pouvoir avoir accès en temps réel à un ensemble de variables caractérisant le comportement des différentes composantes de l'écosystème permet de suivre sa réaction et ses facultés d'adaptation face à ces contraintes. Les premières résultats d'une expérience sur du froment montrent qu'il aura une phénologie plus précoce et que les rendements seront impactés par les sécheresses édaphiques de fin de culture.

\section{Quel type d'écosystème sur nos terres?}

De manière générale, les forêts représentent un puits de gaz à effet de serre (piège à carbone) tandis que l'agriculture constitue une source à cause des émissions de méthane et de protoxyde d'azote. Par conséquent, la première action pour renforcer le rôle de nos écosystèmes terrestres dans la lutte contre l'effet de serre est d'augmenter la surface dédiée aux forêts, par arrêt du déboisement et par (re)forestation. Il faut toutefois être prudent, car toutes les terres ne peuvent pas accueillir des forêts (e.g. les déserts) et nous devons garder assez de terres arables pour nourrir l'humanité et conserver une variété de biotopes suffisante pour préserver la biodiversité. Cette solution n'est de toute façon que partielle ${ }^{[11]}$ car elle permettrait un stockage additionnel de seulement $11 \mathrm{GtCO}_{2} / \mathrm{an}^{[12]}$ ce qui ferait passer l'absorption de $\mathrm{CO}_{2}$ par les écosystèmes de 29 à $58 \%$ des émissions anthropogéniques de $\mathrm{CO}_{2}$ seulement et cela uniquement jusqu'à 2030. Il faut donc trouver des pratiques complémentaires qui correspondent à une gestion qui stocke plus de carbone à très long terme dans les sols à laquelle il faudra ajouter des actions tendant à réduire les émissions agricoles de méthane et de protoxyde d'azote. La problématique est donc complexe et les solutions ne sont pas simples. 


\section{Une gestion agricole et forestière pour atténuer le changement climatique}

Il n'y a pas encore d'accord unanime sur le choix d'itinéraires techniques qui permettraient d'atténuer le changement climatique tout en préservant les autres services écosystémiques. Toutefois, nous sommes en mesure de donner quelques éléments qui permettront aux gestionnaires agricoles et forestiers d'orienter leurs pratiques dans la bonne direction.

En ce qui concerne les grandes cultures, la diminution de l'emploi des fertilisants azotés d'origine minérale permet de réduire les émissions d'azote. Il a été démontré ${ }^{[6]}$ que $3 \%$ de l'azote présent dans ces fertilisants se retrouvait au final dans l'atmosphère sous forme de protoxyde d'azote. Réduire autant que possible l'utilisation de ces fertilisants azotés ou utiliser des solutions alternatives tout en gardant des productions suffisantes pour nos besoins est donc essentiel.

Nous devons également favoriser les pratiques agricoles visant à maximiser la quantité de carbone qui reste dans le sol après l'exploitation ${ }^{[13,14]}$. Par exemple, nous pouvons encourager le choix d'espèces avec des racines profondes, le retour des résidus de cultures dans le sol, l'agroforesterie avec une grande masse de racines venant des arbres ou encore l'incorporation dans le sol de biocharbon qui ne se décompose que très lentement. Là où l'eau est limitante, l'irrigation peut aussi améliorer le développement racinaire. Enfin, l'utilisation d'une culture intermédiaire à la place d'un sol nu pendant les périodes non productives permet d'avoir des entrées supplémentaires de carbone dans le sol et d'éviter le lessivage de l'azote du sol.

Une autre solution envisagée pour augmenter le stockage de carbone dans les sols agricoles est la réduction de la profondeur de labour. En effet, lorsque les couches de sol plus profondes ne sont pas remises en surface, on évite leur exposition au soleil et aux précipitations qui favorisent la décomposition de la matière organique ${ }^{[15]}$. Cependant, cette technique de travail superficiel est antagoniste avec un enfouissement profond des résidus de culture qui fixe le carbone de façon plus stable et empêche la décomposition de la composante azotée des résidus (empêche l'émission de $\mathrm{N}_{2} \mathrm{O}$ ) ${ }^{[16]}$. Son efficacité à long-terme n'a d'ailleurs pas encore pu être démontrée.

La sylviculture quant à elle doit être établie pour garder des forêts vigoureuses qui photosynthétisent le $\mathrm{CO}_{2}$. Pour cela, il est souhaitable que les gestionnaires fassent réaliser des éclaircies régulières mais d'intensité limitée. D'une part, il faut permettre à la lumière de pénétrer dans le couvert pour favoriser la pousse des jeunes arbres en sous-bois (régénération naturelle qui permet de maintenir de façon continue l'assimilation de carbone) mais d'autre part, il faut garder un certain ombrage du sol puisque lorsque le sol est éclairé, il est susceptible d'être exposé à des températures et contenus en eau plus élevés, ce qui favorise la décomposition de sa matière organique (production de $\mathrm{CO}_{2}$, diminution du stock de carbone dans le sol ${ }^{[17]}$. Il est dès lors recommandé d'établir des parcelles avec des arbres d'âge varié et de prélever certains individus de façon régulière. Le choix d'espèces doit aussi s'orienter vers celles à racines profondes permettant une meilleure résistance aux sécheresses et aux tempêtes.

Pour l'élevage, et si on considère uniquement le bilan GHG comme critère d'évaluation, il faudrait favoriser les non-ruminants comme le porc ou le poulet par rapport aux vaches, 
moutons ou chèvres qui produisent beaucoup plus de méthane. De plus, il est préférable d'opter pour des vaches laitières par rapport aux viandeuses puisque la quantité de méthane émis par $\mathrm{kg}$ de protéine est sensiblement moins importante pour le lait que pour la viande ${ }^{[18]}$. Enfin, il est préférable, de nourrir un troupeau à l'herbe en maintenant un nombre d'animaux par unité de surface compatible avec la capacité d'absorption de carbone de la prairie. Cela évite de devoir dédier des surfaces terrestres à la production d'aliments pour animaux (alors qu'elles pourraient accueillir des forêts qui stockeraient plus de carbone) et transporter ces aliments en utilisant du carbone fossile.

Toutes ces propositions pourraient dans une première approximation réduire la concentration atmosphérique de GES de l'équivalent de $23 \mathrm{Gt} \mathrm{CO}_{2} /$ an jusqu'en 2030 (les émissions de $\mathrm{CH}_{4}$ et $\mathrm{N}_{2} \mathrm{O}$ sont transformées en équivalent $\mathrm{CO}_{2}$ au regard de leur effet de forçage radiatif). Cette valeur sera déjà considérable mais encore beaucoup trop petite pour rester sur une tendance suffisante pour atteindre l'objectif d'une hausse maximum de $2^{\circ} \mathrm{C}$ en 2100 (accord de Paris). Cette quantité ne couvrirait en fait que 37\% de nos émissions de GES. Pour améliorer le rôle d'atténuateur d'effet de serre de l'agriculture et des forêts, la recherche doit donc mettre en avant des solutions complémentaires (utilisation d'énergie d'origine non fossile). Reste donc maintenant à les peaufiner et à ce qu'elles soient soutenues par les pouvoirs publics.

\section{Références}

${ }^{1}$ IPCC, 2014: Climate Change 2014: Synthesis Report. Contribution of Working Groups I, II and III to the Fifth Assessment Report of the Intergovernmental Panel on Climate Change [Core Writing Team, R.K. Pachauri and L.A. Meyer (eds.)]. IPCC, Geneva, Switzerland, 151 pp.

${ }^{2}$ L. Perugini et al. (2017) Biophysical effects on temperature and precipitation due to land cover change, Environ. Res. Lett., 12, 053002

${ }^{3}$ IPBES (2019) Summary for policymakers of the global assessment report on biodiversity and ecosystem services of the Intergovernmental Science-Policy Platform on Biodiversity and Ecosystem Services

${ }^{4}$ Global Carbon Project (2018): https://www.globalcarbonproject.org/carbonbudget

${ }^{5}$ The Global Methane Budget (2016): https://www.globalcarbonproject.org/methanebudget

${ }^{6}$ A. Syakila \& C. Kroeze (2011) The global nitrous oxide budget revisited, Greenhouse Gas Measurement and Management, 1:1, 17-26

${ }^{7}$ M. Aubinet,T. Vesala and D. Papale (2012), Eddy covariance: a practical guide to measurement and data analysis, Springer, Dordrecht, New York.

${ }^{8}$ https://www.icos-ri.eu

${ }^{9} \mathrm{http}: / /$ www.icos-belgium.be 
${ }^{10}$ P. Buysse et al. (2017), Carbon budget measurement over 12 years at a crop production site in the silty-loam region in Belgium. Agric. For. Meteorol. 246, 241-255.

${ }^{11}$ D. Baldocchi \& J. Penuelas (2019) The physics and ecology of mining carbon dioxide from the atmosphere by ecosystems, Global Change Biology, Volume 25, Issue 4, Pages 1191-1197

${ }^{12}$ B.W. Griscom et al. (2017) Nature climate solutions, PNAS, Volume 114 $\mathrm{n}^{\circ}$ 44, Pages 11645-11650.

${ }^{13}$ S. Pellerin et al. (2019) Stocker du carbone dans les sols français, Quel potentiel au regard de l'objectif 4 pour 1000 et à quel coût ? Synthèse du rapport d'étude, INRA (France), $114 \mathrm{p}$.

${ }^{14}$ K. Paustian et al. (2016), Climate-smart soils, Nature. doi:10.1038/nature 17174

${ }^{15}$ N.R. Haddaway et al. (2017) How does tillage intensity affect soil organic carbon? A systematic review, Environmental Evidence

${ }^{16}$ M. Lognoul et al. (2017) Impact of tillage on greenhouse gas emissions by an agricultural crop and dynamics of $\mathrm{N}_{2} \mathrm{O}$ fluxes: Insights from automated closed chamber measurements, Soil and Tillage Research, Volume 167, Pages 80-89

${ }^{17}$ V. Coletta et al. (2017) Short-time effect of harvesting methods on soil respiration dynamics in a beech forest in southern Mediterranean Italy, IForest, Volume 10, Issue 3, Pages 645-651

${ }^{18}$ C. Opio et al. (2013) Greenhouse Gas Emissions from Ruminant Supply Chains - a Global Life Cycle Assessment, Report of the Food and Agriculture Organization of the United Nations (FAO) 